EXTENDED REPORT

\title{
Quantification of retinal nerve fiber layer thickness reduction associated with a relative afferent pupillary defect in asymmetric glaucoma
}

\author{
Yasuko Tatsumi, Makoto Nakamura, Miyuki Fujioka, Yoriko Nakanishi, Azusa Kusuhara, Hidetaka \\ Maeda, Akira Negi
}

Br J Ophthalmol 2007;91:633-637. doi: 10.1136/bjo.2006.105494

See end of article for authors' affiliations

.....................

Correspondence to: Makoto Nakamura, Department of Organs Therapeutics, Division of Ophthalmology, Kobe University Graduate School of Medicine, 7-5-2 Kusunoki-cho, Chuo-ku, Kobe, 650-0017, Japan; manakamu@ med.kobe-u.ac.jp

Accepted 5 October 2006 Published Online First 18 October 2006

\begin{abstract}
Aim: The relative afferent pupillary defect (RAPD) is an important clinical sign of asymmetrical retinal ganglion cell and axonal damage. Although glaucoma essentially affects bilateral eyes, a subset of patients manifests asymmetrical glaucomatous optic neuropathy (GON), which exhibits an RPAD in the more advanced eyes. However, the degree to which axonal loss occurs before an RAPD is clinically detectable has not been substantiated. The purpose of this study is to assess the relationship between the depth of a clinically detectable RAPD and the reduction ratio of retinal nerve fiber layer (RNFL) thickness in the more advanced eyes relative to that in the contralateral less advanced eyes of patients with asymmetrical GON.

Methods: Enrolled were 29 consecutive glaucoma patients with the clinically detectable RAPD. An RAPD was quantified by placing log-scaled neutral density filters over the less advanced eyes while performing the swinging flashlight test. Average RNFL thickness was determined using the Fast RNFL thickness programme of optical coherence tomography 3000. Correlation coefficient and Linear regression analyses were used in assessing the relationship between the RAPD and the ratio of RNFL thickness in the more advanced eyes relative to that in the less advanced.

Results: RAPD ranged from 0.6 to $2.4 \mathrm{log}$ units. The log-scaled RAPD had a statistically significantly inversed correlation with the average RNFL thickness ratio $\left(r_{s}=-0.729, p<0.0001\right)$. Linear regression analysis found an equation that the average RNFL thickness ratio in the more affected eyes relative to that in the less advanced $(\%)=(0.827-0.169 \times \operatorname{RAPD}(\log$ units $)) \times 100\left(R^{2}=0.557, \mathrm{p}<0.0001\right)$.

Conclusions: When an RAPD is clinically detected, the RNFL thickness in the more advanced eyes was in average reduced to about $73 \%$ of that in the less advanced.
\end{abstract}

$\mathrm{T}$ he relative afferent pupillary defect (RAPD) is an asymmetry in the pupillary light response and an important objective parameter for quantifying the loss of neuronal function in asymmetric optic nerve and retinal disorders. ${ }^{1}$ Glaucoma is characterised by progressive loss of retinal ganglion cells and their axons with the optic disc excavation and retinal nerve fiber layer (RNFL) defect, which is now recognised as glaucomatous optic neuropathy (GON). Although GON is essentially a bilateral disease, a subset of patients with GON is unilaterally or asymmetrically affected. For instance, Krupin et al recently reported that $27.9 \%$ of patients with open-angle glaucoma were unilaterally involved. ${ }^{2}$ Patients with such an asymmetric glaucoma show an RAPD in the more advanced eyes. $^{3-5}$ However, the degree to which axonal loss occurs in patients with asymmetric GON before an RAPD is clinically detectable is still a matter of interest.

Optical coherence tomography (OCT) is a near infrared, lowcoherent illumination-assisted, non-invasive technique, which provides cross-sectional imaging of the layered structure of the retina with axial resolution of $10 \mu \mathrm{m} .{ }^{6}$ OCT is useful not only for assessment of macular thickness in retinal diseases ${ }^{7}$ but also for estimation of RNFL thickness reduction in glaucoma ${ }^{8}$ as well as other optic nerve disorders. ${ }^{910}$ In our studies, OCT successfully detected the characteristic pattern of RNFL reduction in eyes with band atrophy of the optic nerves due to chiasmal compression and in those with optic tract syndrome. ${ }^{9}{ }^{10}$ On the other hand, we recently reported that an RAPD was clinically detectable in patients with a variety of unilateral optic nerve disorders other than GON when RNFL thickness in the affected eyes was reduced to approximately $75 \%$ of that in the contralateral control eyes. ${ }^{11}$

The purpose of this study is to assess the relationship between the depth of clinically detectable RAPD and the ratio of OCT-estimated RNFL thickness in the more advanced eyes relative to that in the contralateral less advanced eyes of patients with asymmetrical GON.

\section{PATIENTS AND METHODS}

This study followed the principles of the Declaration of Helsinki with the approval of the Medical Ethical Committee of the Kobe University Graduate School of Medicine. Written informed consent was obtained from each participant.

Enrolled were 29 consecutive glaucoma patients (19 males and 10 females) with a clinically detectable RAPD, who regularly visited Glaucoma Clinic in the Kobe University Hospital between February and September 2005. The mean \pm SD age at test was $52.0 \pm 18.0$, ranging from 15 to 82 years.

All subjects underwent full clinical ophthalmologic evaluation including Landolt visual acuity testing, intraocular pressure measurement, slit-lamp stereo biomicroscopy, and indirect ophthalmoscopy. Diagnosis of glaucoma was based on glaucomatous visual field loss with accompanying optic nerve abnormality. The type of glaucoma was consisted of primary open-angle glaucoma in 23 patients, developmental glaucoma

Abbreviations: GON, glaucomatous optic neuropathy; $O C T$, optical coherence tomography; RAPD, relative afferent pupillary defect; RNFL, retinal nerve fiber layer 
in four patients, chronic angle-closure glaucoma in one, and steroid-induced glaucoma in one. Nine patients had asymmetric bilateral glaucoma, while the remaining 20 patients had unilateral glaucoma. All patients were phakic.

Patients were excluded who had a history of blunt ocular injury, severe uveitis, exfoliation glaucoma, diabetes, intraocular surgery, and laser treatment that can affect pupillary light reaction. Additional exclusion criteria included refractive error higher than +3.00 or lower than -6.00 diopters, poor visual acuity not enough to get reliable data in OCT and visual field examinations, and media opacities. Patients who had cataract with grade II or more nuclear sclerosis were also excluded, since dense cataract is known to produce an RAPD in the contralateral eyes. ${ }^{12}{ }^{13}$

Best-corrected visual acuity was measured at $5 \mathrm{~m}$ distance using standard Landolt eye chart (K-3400, INAMI, Japan) and converted in a logarithm scale in statistical analysis.

Visual field examination with Humphrey Field Analyzer using SITA standard 30-2 programme was performed within 3 months when an RAPD was quantified as described in detail below. Subjects were excluded if fixation loss, false-positive errors, or false-negative errors was greater than 30\%. Mean deviation was used as a parameter of the visual field loss evaluated in this study. The criteria proposed by Anderson and Patella $^{14}$ were used to judge the glaucomatous visual field damage. In this study, we defined unilateral glaucoma when the visual filed in the less advanced eyes was normal with the standard automated perimetry.

An RAPD was quantified by performing the swinging flashlight test by placing the neutral density filters over the less advanced eye essentially according to previous reports. ${ }^{13-}$ ${ }^{511}$ In brief, patients in a sitting position were instructed to fixate on a distant target in a mesopic room for $60 \mathrm{~s}$. Then each eye was illuminated for $3 \mathrm{~s}$ using an indirect ophthalmoscope with the visual angle of the light source being 30 degrees. The density of the neutral density filters was incremented in a 0.3log-unit step-wise fashion until the RAPD was undetectable.

The RNFL thickness around the optic disc was determined using OCT 3000 (v 4.2.4, Zeiss-Humphrey, Dublin, California, USA) as reported previously. ${ }^{8-11}$ Briefly, three circular scans, each $3.4 \mathrm{~mm}$ in diameter, centered on the optic disc were obtained after pupillary dilatation, which was averaged by the Fast RNFL thickness (3.4) programme. All measurements were repeated until signal strength of image exceeded 7 (maximum 10) to obtain high accuracy of image. Average RNFL thickness of the entire circumference of the optic disc and quadrant RNFL thickness were obtained.

Statistical analysis was performed by Stat View version 5.0 software (SAS Institute, Cary, North Carolina, USA). The comparison of two independent continuous variables was made with unpaired t-test. Correlation coefficient and linear regression were used in assessing the relationship between RAPD and following parameters: (1) the ratio of RNFL thickness in the more affected eyes relative to that in the less affected; (2) the inter-eye difference of mean deviation value. A p Value of less than 0.05 was considered statistically significant.

\section{RESULTS}

The demographic data of subjects is summarised in table 1 .

The mean refractive error \pm SD was $-1.65 \pm 2.55$ diopters in the less advanced eyes and $-1.71 \pm 2.65$ diopters in the more advanced, which was not significantly different $(p=0.9399)$. The log-converted best-corrected visual acuity ranged from 0.18 to -0.15 in the less advanced eyes and from 0.30 to -0.52 in the more affected eyes $(\mathrm{p}=0.0801)$.

The mean deviation value was $-2.79 \pm 3.97 \mathrm{~dB}$ in the less advanced eyes and $-20.23 \pm 7.16 \mathrm{~dB}$ in the more advanced eyes. The difference was statistically significant $(p<0.0001)$.
Average RNFL thickness was $83.93 \pm 17.98 \mu \mathrm{m}$ in the less advanced eyes and $50.60 \pm 14.67 \mu \mathrm{m}$ in the more advanced. The latter was significantly thinner than the former $(p<0.0001)$. Quadrant RNFL thickness of the less advanced eyes and the more advanced eyes was $100.52 \pm 25.65$ and $58.79 \pm 20.70$ in the superior quadrant $(\mathrm{p}<0.0001), 103.69 \pm 27.67$ and $48.48 \pm 20.20$ $(\mathrm{p}<0.0001)$ in the inferior, 67.62 \pm 15.13 and $46.62 \pm 15.52$ in the temporal $(\mathrm{p}<0.0001)$, and $63.38 \pm 19.84$ and $50.60 \pm 14.67 \mu \mathrm{m}$ in the nasal $(\mathrm{p}=0.0072)$, respectively (raw data not shown).

RAPD ranged from 0.6 to 2.4 log units. Spearman's correlation coefficients between RAPD and the other tested parameters were as follows: the inter-eye mean deviation difference, $r_{s}=0.726(p=0.0001)$; the average RNFL thickness ratio in the more advanced eyes relative to the less advanced, $\mathrm{r}_{\mathrm{s}}=-0.729(\mathrm{p}<0.0001)$; the quadrant RNFL thickness ratio, $\mathrm{r}_{\mathrm{s}}=-0.569(\mathrm{p}=0.0013)$ in the superior quadrant, $\mathrm{r}_{\mathrm{s}}=-0.734$ $(\mathrm{p}<0.0001)$ in the inferior, $\mathrm{r}_{\mathrm{s}}=-0.511(\mathrm{p}=0.0037)$ in the temporal, and $\mathrm{r}_{\mathrm{s}}=-0.386(\mathrm{p}=0.0259)$ in the nasal, respectively.

Using linear regression analysis, an equation was found that the reduction ratio of average RNFL thickness in the more affected eyes relative to that in the less advanced (\%) $=\{0.827-0.217 \times$ RAPD $\quad(\log$ units $)\} \times 100 \quad\left(R^{2}=0.557\right.$, $\mathrm{p}<0.000 \mathrm{l}$; fig 1). On the other hand, there was also a significant association between the depth of the RAPD and the inter-eye mean deviation difference, quantified by an equation:

$\mathrm{dB}=6.57 \mathrm{l}+8.615 \times \mathrm{RAPD}(\log$ units $)\left(\mathrm{R}^{2}=0.613, \mathrm{p}<0.0001 ;\right.$ fig 2).

Where $\mathrm{dB}$ is the mean deviation difference.

\section{DISCUSSION}

In the current study, we have demonstrated that the RAPD was inversely associated with the rate of average RNFL thickness in patients with asymmetric GON and was clinically detectable when average RNFL thickness around the optic disc was reduced by $27 \%$ in the more affected eyes compared with the less affected. This estimation was extrapolated from the simple liner regression line when $\mathrm{x}=0.6 \log$ unit was applied (fig 1 ).

The minimal density of an RAPD that we could identify in this study was 0.3-log unit. However, some claimed it often difficult to reliably quantify such a small RAPD as 0.3-log unit or less by the swinging flashlight test. ${ }^{15}$ Thus, we chose the 0.6$\log$ unit as the clinically detectable minimal depth of an RAPD in this study. The association may become stronger if more sophisticated tools are applied to quantify an RAPD such as infrared pupillography. However, we followed the current protocol because we wanted to know to what degree an RAPD detected by the routine swinging flashlight test correlates with the structural change of retinal nerve fiber caused by GON.

In a prior paper, we demonstrated using a similar experimental design that the swinging flash light test could detect an RAPD in patients with unilateral optic neuropathy with a variety of etiologies when the RNFL thickness was reduced by at least $25 \%$ as compared with the contralateral control eyes. ${ }^{11}$ Kerrison et al previously showed that in rhesus monkeys, which underwent laser ablation of macular tissue, an RAPD developed when approximately $25 \%$ to $50 \%$ of retinal ganglion cells were histologically lost. ${ }^{16}$ Lagreze and Kardon correlated the RAPD with the ganglion cell loss estimated from visual field defect. ${ }^{17}$ According to this study, regression analysis between the RAPD and the ganglion cell loss determined from the pattern deviation with $5 \%$ probability level had $\mathrm{R}^{2}=0.49(\mathrm{P}<0.0001)$. The current estimation of the RNFL thickness ratio associated with the development of the clinically detectable RAPD was in good agreement with the above biometrical, histological, and functional assessment of the axonal loss related to the RAPD. 


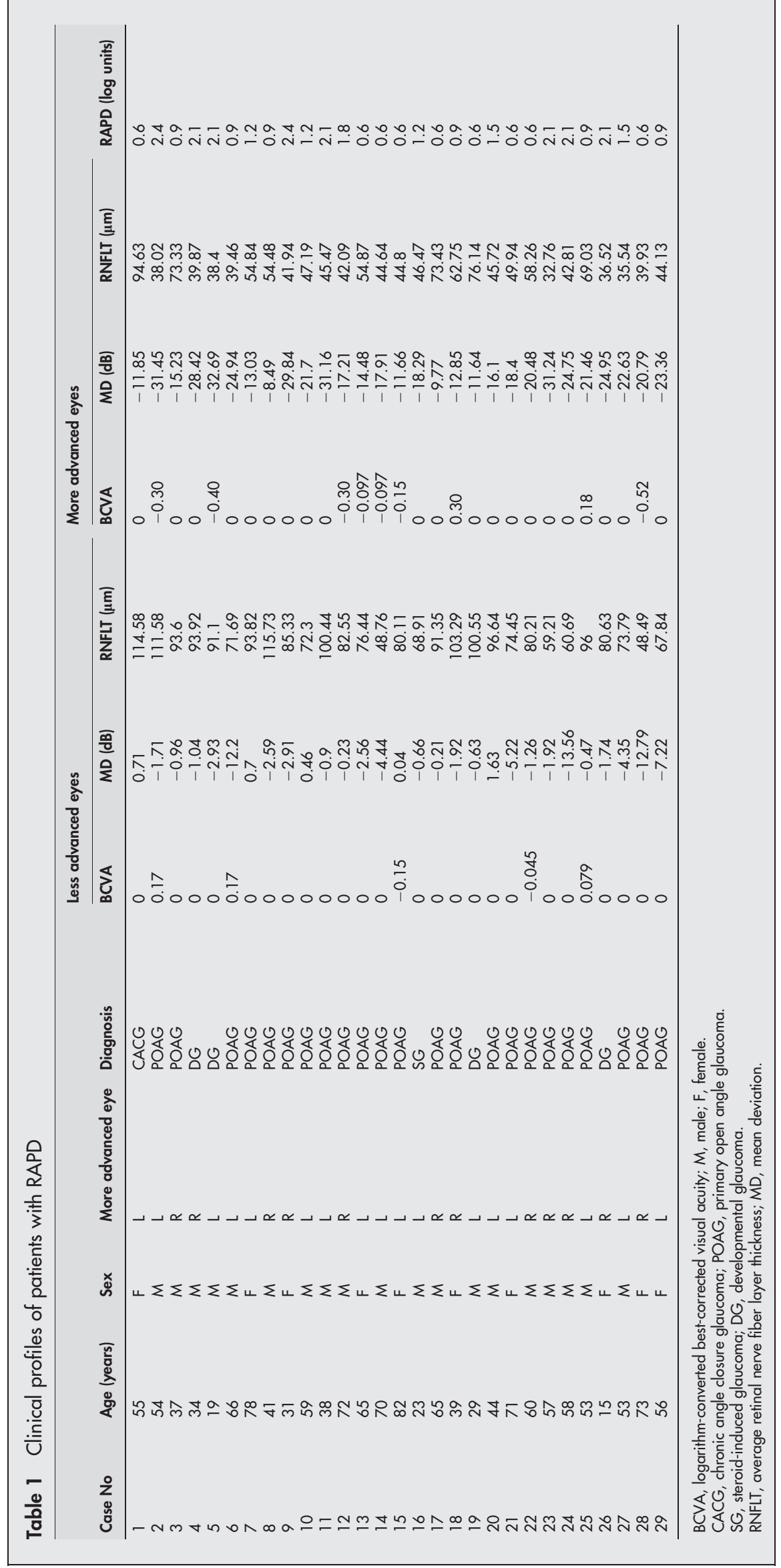




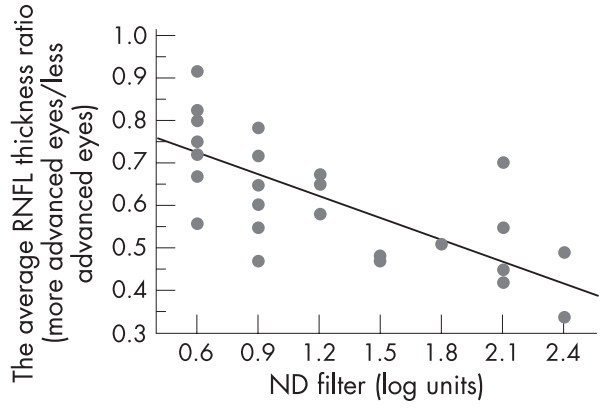

Figure 1 Linear regression analysis of the relationship between the average RNFL thickness ratio in the more advanced eyes to the less advanced measured by optical coherence tomography and the depth of relative afferent pupillary defect determined by the log-units of the neutral density filters. $R^{2}=0.557, p<0.0001$.

In the current study, the RAPD also correlated with the quadrant RNFL thickness ratio. The Spearman's correlation coefficient was strongest in the inferior quadrant, followed by the superior, temporal, and nasal quadrant in a descending order. It is not surprising that the RNFL thickness ratio in the inferior and superior quadrants had better correlation with the depth of an RAPD than that in the temporal or nasal quadrant, because vertical segments of the optic disc are well known to be earlier and more severely damaged than horizontal segments in eyes with GON. In this study, visual field defect was superiorly dominant in 14 eyes $(36.8 \%)$ and inferiorly dominant in four eyes $(10.5 \%)$, whereas the remaining 20 eyes $(52.6 \%)$ did not show significant difference of superior and inferior visual field defects. This may be the reason why the inferior quadrant RNFL thickness ratio had a closer association with the depth of the RAPD than the superior quadrant.

The present and previous studies further indicate that substantial retinal ganglion cell damage precedes the development of the clinically detectable RAPD. This threshold effect regarding the relationship between the RNFL thickness reduction and the RAPD development may be accounted for by nerve fiber redundancy in the anterior visual pathways.

The degree of retinal nerve fiber loss that develops an RAPD in eyes with optic neuropathy was a etiology-independent as far as the swinging flash light test was used. As mentioned above, we recently evaluated the association between the RNFL thickness reduction determined with the OCT 3000 and the log-scaled density of an RAPD in 20 patients with unilateral optic atrophy other than GON. ${ }^{11}$ Aetiology of these patients included anterior and posterior ischemic optic neuropathy, compressive optic neuropathy, and traumatic optic neuropathy. In that study, the correlation coefficient $\mathrm{R}^{2}$ was 0.48 $(p=0.0007)$ between the two variables and the estimated RNFL thickness reduction was $23 \%$ when the $0.6-\log$ unit RAPD was detected, which was closely similar to the current estimation in patients with asymmetric GON.

RNFL is largely composed of magnocellular ganglion cell axons, which essentially correspond to the superior, inferior, and nasal quadrants of the parapapillary RNFL distribution. In contrast, the parvocelluar ganglion cell axons, which are the major component of the papillo-macular bundle and correspond to the temporal quadrant, are mainly responsible to the light reflex. Whether GON predominantly affects the magnocellular axons compared with the parvocellular axons has been a matter of controversy. Previous histological assessments claimed the vulnerability of the magnocelular axons in eyes with GON, ${ }^{18}$ whereas psychophysical and electrophysiological studies showed the early damage of the parvocellular axons. ${ }^{19}$

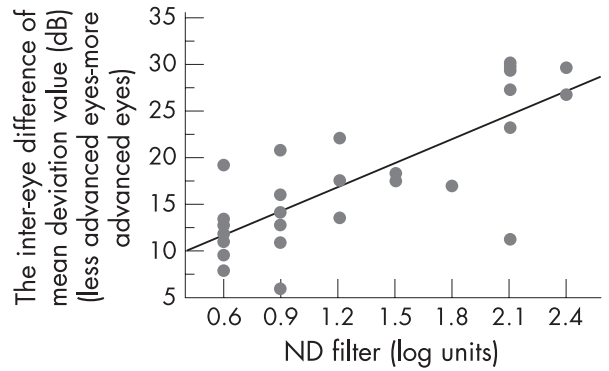

Figure 2 Linear regression analysis of the relationship between the intereye difference of mean deviation value and the depth of relative afferent pupillary defect determined by the log-units of the neutral density filters. $\mathrm{R}^{2}=0.613, \mathrm{p}<0.0001$

The similar association between the RAPD development and the RNFL thickness reduction in eyes with GON and in those with other types of optic neuropathies may provide an additional piece of evidence that the GON affects the ganglion cell axons in a similar way to the other types of optic neuropathies; that is, there may be no preponderance of ganglion cell types affected by GON. On the other hand, the closer association of the quadrant RNFL thickness ratio in the vertical quadrants than in the temporal quadrant with the depth of RAPD may reflect the vulnerability of the magnocellular ganglion cells compared with the parvocellular in eyes with GON. To confirm this issue, more meticulous comparisons between the quadrant or segment RNFL thickness reduction and the RAPD using an infrared pupillography should be made, because the precise retinotopic mapping corresponding to the RAPD development as well as the more quantitative evaluation of an RAPD are required.

In this study, the log-scaled RAPD also had a significant correlation with the inter-eye mean deviation difference in patients with asymmetric glaucoma. The relationship between the depth of an RAPD and the magnitude of the visual field has been demonstrated. In previous studies, the patients with bilateral glaucoma developed an RAPD (0.3-log unit) when the inter-eye difference in visual field sensitivity was at least $13 \%$ on the program 32 of the OCTOPUS 2000 perimeter. ${ }^{5}$ Quigley et al reported that at about $20 \%$ of retinal ganglion cells were lost when a $5 \mathrm{~dB}$ sensitivity loss was detected at the corresponding points within the central 30 degree of the retina. ${ }^{20}$ The present estimation of about $27 \%$ loss of retinal nerve fibers and about $12 \mathrm{~dB}$ sensitivity loss associated with the development of the clinically detectable RAPD with 0.6-log unit further corroborates the previous observations.

There is another clinical importance in the current study. Visual field progression in patients who have open-angle glaucoma and asymmetrical visual field damage are known to be faster in the initially more affected eyes $(25 \%$ at 5 years) than in the less affected eyes $(7.2 \%$ at 5 years). The risk of progression increases as the difference of initial visual field loss increases..$^{21}$ Thus, the existence of an RAPD may be indicative of not only the substantial difference of the RNFL thickness between eyes but also the risk of the further visual field progression in the eyes with the RAPD.

In conclusion, the log-scaled RAPD quantified by the swinging flash light test with graded neutral density filters was associated with the structural loss of retinal nerve fibers and/or ganglion cells. The RAPD was clinically detectable when average RNFL thickness centered on the optic disc in the more advanced eye was reduced to about $73 \%$ of the contralateral less advanced eyes. 


\section{Authors' affiliations}

Y Tatsumi, M Nakamura, M Füioka, Y Nakanishi, A Kusuhara, H Maeda, A Negi, Department of Organs Therapeutics, Division of Ophthalmology, Kobe University Graduate School of Medicine, Kobe, Japan

Funding: This study was supported in part by Grant-in-Aid No. 16390499 (AN, MN) and No. 17591835 (MN) from the Ministry of Education, Culture, Sports, Science and Technology of the Japanese Government, and by Suda Memorial Foundation for Glaucoma Research (MN). MN is a recipient of the 12th ROHTO Award.

Competing interests: None.

\section{REFERENCES}

1 Digre KB. Principles and techniques of examination of the pupils, accommodation, and lacrimation. In:Miller NR, Newman NJ.Walsh and Hoyt's Clinical Neuro-Ophthalmology, 6th edn. Baltimore: Lippincott Williams \& Wilkins, 2005:715-737.

2 Krupin T, Liebmann JM, Greenfield DS, et al. The low-pressure glaucoma treatment study (LoGTS). Ophthalmology 2005;1 12:376-385.

3 Kaback MB, Burde RM, Becker B. Relative afferent pupillary defect in glaucoma. Am J Ophthalmol 1976;81:462-468.

4 Prywes AS. Unilateral afferent pupillary defects in asymmetric glaucoma. Arch Ophthalmol 1976;94:1286-1288.

5 Brown RH, Zilis JD, Lynch MG. The afferent pupillary defect in asymmetric glaucoma. Arch Ophthalmol 1987;105:1540-1543.

6 Fujimoto JG, Hee MR, Huang D, et al. Principles of Optical Coherence Tomography. In:Schuman JS, Puliafito CA, Fujimoto JG.Optical coherence tomography of ocular diseases, 2nd edn. Thorofare: Slack Inc, 2004:3-19.

7 Jaffe GJ, Caprioli J. Optical coherence tomoglaphy to detect and manage retinal disease and glaucoma. Am J Ophthalmol 2004;137:156-169.

8 Kanamori A, Nakamura M, Escano MFT, et al. Evaluation of the glaucomatous damage on retinal nerve fiber layer thickness measured by optical coherence tomography. Am J Ophthalmol 2003;135:513-520.
9 Kanamori A, Nakamura M, Matsui N, et al. Optical coherence tomography defects characteristic retinal nerve fiber layer thickness corresponding to band atrophy of the optic discs. Ophthalmology 2004; 111:2278-2283.

10 Tatsumi Y, Kanamori A, Kusuhara A, et al. Retinal nerve fiber layer thickness in optic tract syndrome. Jpn J Ophthalmol 2005;49:294-296.

11 Nakanishi Y, Nakamura M, Tatsusmi Y, et al. Quantification of retinal nerve fibe layer thickness reduction associated with a relative afferent pupillary defect. Graefe's Arch Clin Exp Ophthalmol 2006;244:1480-4.

12 Hwang J, Kim C, Kim J. Relative afferent pupillary defect in patients with asymmetric cataracts. J Cataract Refract Surg 2004;30:132-136.

13 Lam BL, Thompson HS. A unilateral cataract produces a relative afferent pupillary defect in the contralateral eye. Ophthalmology 1990;97:334-338.

14 Anderson DR, Patella VM. Automated static perimetry, 2nd edn., St Louis: Mosby 1999:152-153.

15 Kawasaki A, Moore P, Kardon RH. Long-term fluctuation of relative afferent pupillary defect in subjects with normal visual function. Am J Ophthalmol 1996; 112:875-882.

16 Kerrison JB, Buchanan K, Rosenberg ML, et al. Quantification of optic nerve axon loss associated with a relative afferent pupillary defect in the monkey. Arch Ophthalmol 2001;119:1333-1341.

17 Lagreze WD, Kardon RH. Correlation of relative afferent pupillary defect and estimated retinal ganglion cell loss. Graefe's Arch Clin Exp Ophthalmol 1998;236:401-404.

18 Stamper RL. The effect of glaucoma on central visual function. Trans Am Ophthal Soc 1984;82:792-826.

19 McKendrick AM. Badcock DR. Morgan WH. Psychophysical measurement of neural adaptation abnormalities in magnocellular and parvocellular pathways in glaucoma. Invest Ophthalmol Vis Sci 2004;45:1846-1853.

20 Quigley HA, Dunkelberger GR, Green WR. Retinal ganglion cell atrophy correlated with automated perimetry in human eyes with glaucoma. Am J Ophthalmol 1989;107:453-464.

21 Chen PP, Park RJ. Visual field progression in patients with initially unilateral visual field loss from chronic open-angle glaucoma. Ophthalmology 2000;107:1688-1692. 